\title{
Enhanced expression of PD L1 in cervical intraepithelial neoplasia and cervical cancers
}

\author{
Louisa Mezache $^{1}$, Bernard Paniccia ${ }^{1}$, Angelique Nyinawabera ${ }^{1}$ and Gerard J Nuovo ${ }^{1,2}$ \\ ${ }^{1}$ Phylogeny Inc., Powell, OH, USA and ${ }^{2} \mathrm{OSU}$ Comprehensive Cancer Center, Columbus, OH, USA
}

\begin{abstract}
Programmed death ligand 1 (PD L1) expression can reduce the immune response in both infectious diseases and cancers. We thus examined PD L1 expression in cervical intraepithelial neoplasias (CINs) and cancers since they each reflect infection by human papillomavirus (HPV). PD L1 protein was not evident by immunohistochemistry in histologically normal cervical epithelia (0/55) even when adjacent to CIN or cancer. PD L1 expression was much increased in CINs $(20 / 21=95 \%)$ and cervical squamous cell cancer $(56 / 70=80 \%)$ and localized to the dysplastic/neoplastic squamous cells and mononuclear cells, respectively. There was also a significant increase (each $\boldsymbol{P}<0.001$ ) in PD L1 detection in mononuclear cells when comparing cervical squamous cell cancers to endometrial $(22 / 115=19 \%)$ and ovarian adenocarcinomas $(5 / 40=13 \%)$. Co-expression analyses showed that the primary inflammatory cell that contained PD L1 was the CD8+ lymphocyte that strongly concentrated around the dysplastic CIN cells and nests of invasive squamous cancer cells. These data show that PD L1 is a solid biomarker of productive HPV infection of the cervix and that it is significantly upregulated in both the carcinoma and surrounding inflammatory cells in cervical cancer when compared with other gynecologic malignancies. This suggests that anti-PD L1 therapy may have a role in the treatment of cervical cancer.

Modern Pathology (2015) 28, 1594-1602; doi:10.1038/modpathol.2015.108; published online 25 September 2015
\end{abstract}

Infection by human papillomavirus is the cause of cervical intraepithelial neoplasia (CIN). ${ }^{1-5}$ Viral infection is initiated in the transformation zone of the cervix due to the presence of the target squamous metaplastic cell. ${ }^{5-7}$ The histologic correlates of early productive infection include disorganized squamous cell hyperplasia and the koilocyte, which is a cell toward the surface of the lesion that shows a large, well defined perinuclear halo and nuclear atypia. ${ }^{1-5}$ The koilocyte typically has hundreds of copies of HPV DNA/RNA and is the source of infectious virions that can then be sexually transmitted. ${ }^{1-7}$ High grade CIN, which show a more disorganized hyperplasia and fewer koilocytes compared to CIN 1, contains on average much less HPV DNA per dysplastic cell and may presage cervical cancer. ${ }^{1-7}$ HPV DNA typically is integrated in cervical cancers, which is associated with disruption of the E2 ORF, expression of the oncoproteins E6 and E7, and loss of the late capsid proteins. ${ }^{1-8}$

It is documented that if untreated about one-half of CIN 1 lesions (mild dysplasia) regress. ${ }^{1-5}$ It has been

Correspondence: Dr GJ Nuovo, MD, Molecular Medicine, Ohio State Univ Comprehensive Cancer Center, Phylogeny Lab, 1476 Manning Parkway, Powell, OH 43065, USA.

E-mail: nuovo.1@osu.edu

Received 6 February 2015; revised 21 August 2015; accepted 22 August 2015; published online 25 September 2015 shown that in the majority of CIN 1 lesions that spontaneously regress the viral DNA is no longer detectable by highly sensitive techniques, suggesting immune mediated viral clearance. ${ }^{9,10}$ Such spontaneously regressing lesions do contain higher numbers of CD8 $\mathrm{T}$ cells compared with the persistent lesions. ${ }^{11}$ Also, after cryo or thermal ablation of a CIN lesion, $\sim 85 \%$ of post treatment cervices will be negative for the HPV type that caused the infection. In the cases of recurrent CIN after ablation, the second CIN lesion invariably contains a different HPV type than the primary lesion again suggesting that immune clearance of HPV is both efficient and type specific. ${ }^{12}$ This is the basis of the highly effective HPV vaccines that are directed against the L1 proteins of the most common genital HPV types. ${ }^{13,14}$

Programmed death ligand 1 (PD L1) is a protein that is capable of strongly modulating the adaptive arm of the immune system. ${ }^{15,16}$ PD L1 can bind to its ligands that include PD 1 and CD80 that, in turn, can reduce both the proliferation and activity of the cytotoxic CD8 T cells response to both viral or cancer associated antigens. ${ }^{15,16}$ Increased PD L1 expression may allow viruses to avoid immune surveillance. ${ }^{17}$ Recently, two groups showed that therapy directed against PD L1 can cause a strong anti-tumor response in a wide variety of metastastic cancers. These manuscripts also documented that cancers that did 
respond to the anti-PD L1 therapy showed a strong CD8 T-cell presence in the untreated tumor with concomitant high PD L1 expression as seen by immunohistochemistry. ${ }^{15,16}$

The purpose of this manuscript was to compare the expression of PD L1 in the normal cervix, CIN 1-2, cervical cancer (and other gynecologic cancers including endometrial and ovarian carcinoma), and to correlate this with the concomitant immune response.

\section{Materials and methods}

\section{Patient Selection}

Paraffin embedded, formalin fixed de-identified biopsies were available from the files of Phylogeny (Powell, Ohio) and Enzo Clinical Laboratory (Farmingdale, NY). Available clinical information included the histology diagnosis, Pap smear report, prior cervical pathology, and HPV history. The pathologic diagnosis was confirmed by GJN. We examined a total of 301 biopsies for PD L1 expression that included 55 normal cervical biopsies, 21 CIN1-2 lesions, 70 squamous cell carcinomas of the cervix, 40 ovarian adenocarinomas (all serous cystadenocarcinomas), and 115 endometrial adenocarcinomas FIGO 1 (21cases), FIGO 2 (84 cases), and FIGO 3 (10 cases). The cervical biopsies ranged from $5 \mathrm{~mm}$ to $2 \mathrm{~cm}$. Ten of the 55 normal cervical tissues were from women without evidence of HPV infection on histologic exam who had cervical biopsies for atypical Pap smears, usually ASCUS, that were positive for HPV by Hybrid Capture 2 (Digene, Gaithersburg, MD). It should be stressed that each of these 10 biopsies was considered 'equivocal for dysplasia' and was sent for a second opinion review. The 'negative for CIN' final diagnosis was based on the histologic review and negative HPV in situ hybridization result. The remaining 45 normal cervical tissues were adjacent to either CIN 1-2 (8 cases) or cervical cancer (37 cases).

\section{In Situ Hybridization}

HPV in situ hybridization was done on each of the negative for CIN tissues and CIN lesions using a previously published protocol. ${ }^{18,19}$ The presence of HPV was determined using the PathoGene Alkaline Phosphatase NBT/BCIP human papillomavirus in situ typing assay for tissue sections (Enzo Life Sciences, Farmingdale, NY).

\section{Immunohistochemistry}

Our immunohistochemistry protocol has been previously published. ${ }^{18,19}$ In brief, after using a standard optimization protocol, that included positive controls known to have the target of interest, we tested each tissue for CD8 (Ventana Medical System), PD L1 and PD1 (each from ABCAM). Selected cases that showed strong PD L1 expression and that had sufficient remaining tissue were also tested for CD3, CD68, CD45, CD 117, Ki67, CD45RO, and CD20 (each from Ventana Medical System) as well as RANTES and granyzme B (Enzo Life Sciences). All immunohistochemistry was done using the automated Bond Max system (Leica Biosystems).

\section{Co-Expression Analysis}

Co-expression analyses were done using the Nuance system (CRI) as previously published. ${ }^{19}$ In brief, a given tissue was tested for two different antigens using fast red as the chromogen for one target followed by immunohistochemistry using DAB (brown) as the second chromogen with hematoxylin as the counterstain. The results were then analyzed by the Nuance and InForm systems in which each chromogenic signal is separated and converted to a fluorescence based signal, then mixed to determine what percentage of cells were expressing the two proteins of interest.

\section{Cell Culture}

Our cell culture protocol has been previously published. ${ }^{19}$ We grew SiHa (1 HPV DNA copy/cell), HeLa (20 HPV DNA copies/cell), and Caski (hundreds of HPV DNA copies/cell) in DMEM media until $75 \%$ confluent, then fixed the cells in $10 \%$ buffered formalin overnight, washed the cells several times, then processed them for immunohistochemistry and in situ hybridization analyses.

\section{Statistical Analysis}

The Pearson's $\chi^{2}$ test was done using the InStat Software for Windows (version 3.36) testing the null hypothesis that the probability of the expression of PD L1 in one histologic category (precancer or cancer) was equivalent to the probability in a separate group. The $2 \times 2$ table analysis used the continuity correction and the null hypothesis was rejected if the significance level at 1 d.f. was below $1 \%$.

\section{Results}

\section{Histologic and Viral Characterization of the Lesions}

Serial sections were prepared from each block and the initial histologic diagnosis was confirmed from the hematoxylin and eosin stained recut. None of the 55 cervical tissues deemed negative for CIN showed a signal with any HPV probe. Each of the 21 CIN lesions was positive for HPV DNA and all showed a strong signal indicative of high viral copy number. 

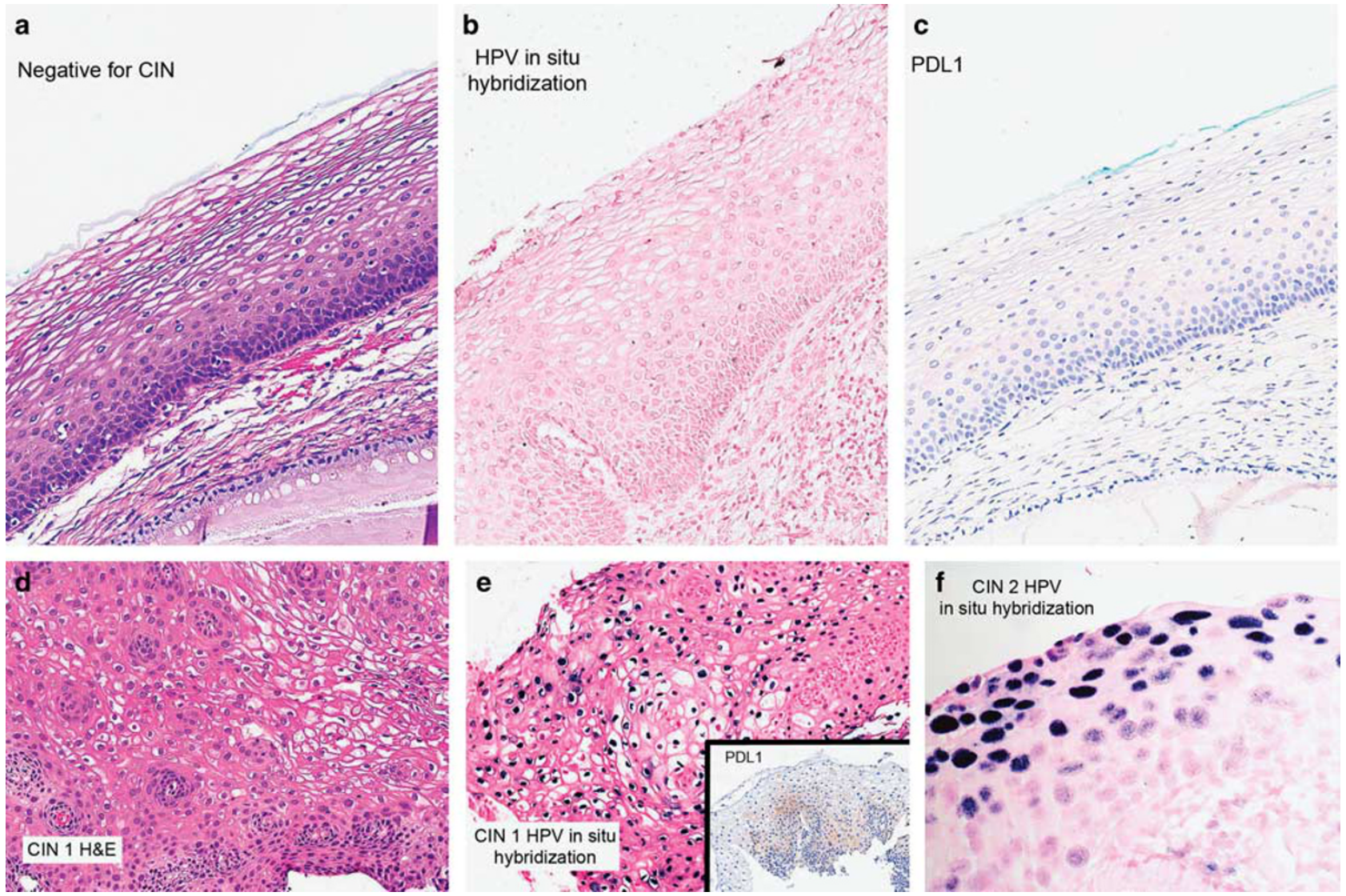

Figure 1 Histologic and viral correlates of cervical pathology. (a) A representative hematoxylin and eosin (H\&E) image of a cervical biopsy at the transformation zone initially deemed to be 'suggestive of CIN 1' due primarily to the presence of many cells with halos. The HPV in situ hybridization test was negative (b). Note that the immunohistochemistry test for PD L1 was also negative in the serial section (c). In comparison, (d) a classic CIN 1; note the increased cell density, irregular cell growth, and variable sizes and shapes to the halos. This tissue was strongly positive for HPV type 51 ((e); the insert shows the positive PD L1 signal toward the base of the lesion. (f) The HPV in situ hybridization for a CIN 2/3 lesion. Note the strong pan-nuclear signal in the surface cells for HPV 16 DNA and the weaker, punctate signal toward the basal zone.

Figure 1 shows representative examples of the histologic and viral in situ results. Fifty of the squamous cell cancers were tested for HPV DNA by in situ hybridization and 42/50 (84\%) were positive.

\section{PD L1 Expression}

Positive controls for PD L1 expression by immunohistochemistry including several melanomas known to show high expression of this protein and negative controls included omission of the primary antibody in positive cases. The controls gave the expected results. Immunohistochemistry was then done on each of the 301 tissues for PD L1. As the positive controls showed that the PD L1 signal could be found in both the cancer and mononuclear cells, which has been reported by others, ${ }^{15,16}$ we decided to separately score both the epithelial and inflammatory cell compartments for signal. Each tissue was given a score of either $0,1+$ (from 1 to $9 \%$ of the target cells + ), 2+ (from 10 to $33 \%$ of the target cells +$)$ or $3+(>33 \%$ of the target cells +$)$. It should be stressed that only tissues that were scored as $2+$ or $3+$ were considered positive so as to highlight cases in which there was abundant PD L1 protein production.

Each of the 18 histologically negative for CIN cervices, which included 8 tissues adjacent to CIN 1-2 lesions were negative for PD L1 in both the squamous cell and mononuclear cell components. Similarly, none of the cervical epithelial tissue (squamous or glandular cell) showed a signal for PD L1 in the 37 histologically normal cervical tissues that were adjacent to cervical squamous cell cancer. Scattered mononuclear cells were positive for PD L1 in the normal cervical tissues that were adjacent to the squamous cell cancers but only in $3 / 37$ cases $(8 \%)$.

Thus, we established that PD L1 was not expressed in either the epithelial or mononuclear compartments in histologically negative for CIN tissues from women who were positive for HPV by hybrid capture even if a CIN 1-2 lesion was present in a different part of the cervix. Similarly, PD L1 was not evident in the normal cervical epithelia adjacent to cervical 
Table 1 Detection of PD L1 in the dysplastic/neoplastic epithelial and inflammatory cells of cervical, endometrial, and ovarian lesions

\begin{tabular}{lcr}
\hline Pathology diagnosis & PD L1 (epithelial cells) ${ }^{\mathrm{a}}$ & PD L1 (mononuclear cells) $^{\mathrm{a}}$ \\
\hline Squamous epithelia of cervix, negative for CIN & No evidence of CIN 0/10 \\
& No evidence of CIN 0/10 & Adjacent to CIN 0/8 \\
& Adjacent to SCC 3/37 & TOTAL 3/55 \\
CIN 1-2 & Adjacent to SCC 0/8 $0 / 37$ & $13 / 21(61 \%)$ \\
Squamous cell cancer-Cervix & TOTAL 0/55 & $56 / 70(80 \%)$ \\
Endometrial adenocarcinoma & $20 / 21(95 \%)$ & $22 / 115(19 \%)$ \\
Ovarian adenocarinoma & $36 / 70(51 \%)$ & $5 / 40(13 \%)$ \\
\hline
\end{tabular}

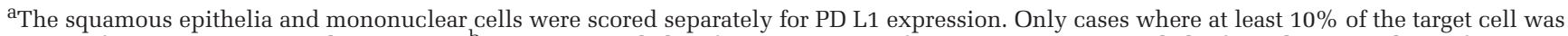
positive for PD L1 were scored as positive. ${ }^{b}$ Squamous epithelia of cervix, negative for CIN = Squamous epithelia from the cervical transformation zone from women with atypical Pap smears and HPV+by hybrid capture that on histologic exam were deemed negative for CIN with a corroborative negative HPV in situ hybridization result. ${ }^{\mathrm{C}} \mathrm{SCC}$, squamous cell carcinoma of the cervix.

cancer. We then compared this data to that found in CIN 1-2 lesions. This data is presented in Table 1. Note that high PD L1 expression was found in 20/21 $(95 \%)$ of the dysplastic epithelial cells of CIN 1-2 lesions. PD L1 was scored as $2-3+$ in the mononuclear cells in 13/21 (61\%) of the CIN 1-2 lesions. Both values were highly significant when compared with the histologically negative for CIN squamous tissue $(P<0.001)$. Figure 2 shows a representative example of PD L1 expression in a CIN 1 lesion. Note that PD L1 was typically found toward the basal zone of the CIN lesion and that most of the mononuclear cells expressing PD L1 were near the interface of the basal epithelia of the CIN lesion and stroma. In comparison, HPV DNA is typically most abundant in the upper layers of a CIN 1-2 lesion. Thus, coexpression of HPV DNA and PD L1 showed that these two targets were in the same areas, but in different cell types (Figure 2).

We next compared the expression of PD L1 in the three most common gynecologic malignancies: cervical (squamous cell), endometrial adenocarcinoma, and ovarian serous cystadenocarcinoma. This data are likewise presented in Table 1 . Note that the expression of PD L1 in both the malignant epithelial and mononuclear cell component was markedly and significantly greater in the squamous cell cervical cancers when compared with the endometrial and ovarian carcinomas $(P$-values all $<0.001)$. Representative data in presented in Figure 3. Note that the strong PD L1 expression in cervical cancers was often found in the epithelial and mononuclear cells at the invading front of the tumor.

Several differences were noted when comparing the PD L1 expression of CIN 1-2 lesions and cervical squamous cell carcinoma. In the abnormal squamous cells, the percentage of cases with high PD L1 expression was less in the squamous cell cancers (51\%) compared with the CIN 1-2 lesions (95\%). However, the mononuclear compartment of CIN 1-2 lesions showed a smaller percentage of PD L1 positive cases $(61 \%)$ compared with the same compartment in squamous cell cancers $(80 \%)$. Another difference was that many mononuclear cells that expressed PD L1 were seen in the nests of invasive squamous cells. However, PD L1 positive mononuclear cells were rarely evident in the epithelial layer of CIN 1-2 (Figures 1 and 2). Further, the number of CD8+ cells around the HPV infected squamous cells tended to be much higher for cervical cancers than for CIN 1-2 lesions (Figures 1 and 2).

It was thus evident that PD L1 expression was much increased in the HPV infected tissues of the cervix, including CINs and invasive squamous cell cancers, compared with both normal cervical tissues and other gynecologic carcinomas. We then addressed the overall immunophenotypic response in the areas of PD L1 positivity in the squamous cell cancers of the cervix. This was done by analyzing serial sections for CD3, CD8, CD20, CD45, CD45RO, CD 117, and CD68 and then doing co-expression analysis as indicated. It was clear from this data that the immune cell type that dominated in the PD L1 positive areas was the CD8+ cytotoxic T-cell (Figure 4). The CD8+ cell was also the most common mononuclear cell to infiltrate the nests of squamous cell cancer. Also note the strong representation of B cells, macrophages, and natural killer (NK) cells in these areas. Given that CD8 and its potential inhibitors, PD L1, were each dominant in both CINs and cervical cancers, the next issue we assessed was the activity of the CD8 cells. In this regard we studied serial sections for RANTES, granzyme B, and Ki67 expression; the latter was shown to be an indicator of activated CD8 cells in a recent study. ${ }^{16}$ We noted that only a small proportion of the CD8 cells in a given area of a cervical cancer expressed RANTES, granzyme B, or were Ki67 positive (Figure 4) suggesting that most of the CD8 cells were in a relatively quiescent state that may be due to the high colocalization with PD L1.

As a primary activator of PD L1 is the protein PD 1, we examined the cervical tissues for PD 1 expression by immunohistochemistry. PD 1 expression indeed closely mirrored PD L1 production in both the CINs and cervical cancers (data not shown) and was rarely noted in the negative for HPV parts of the cervical tissues. The next question we addressed was, which 

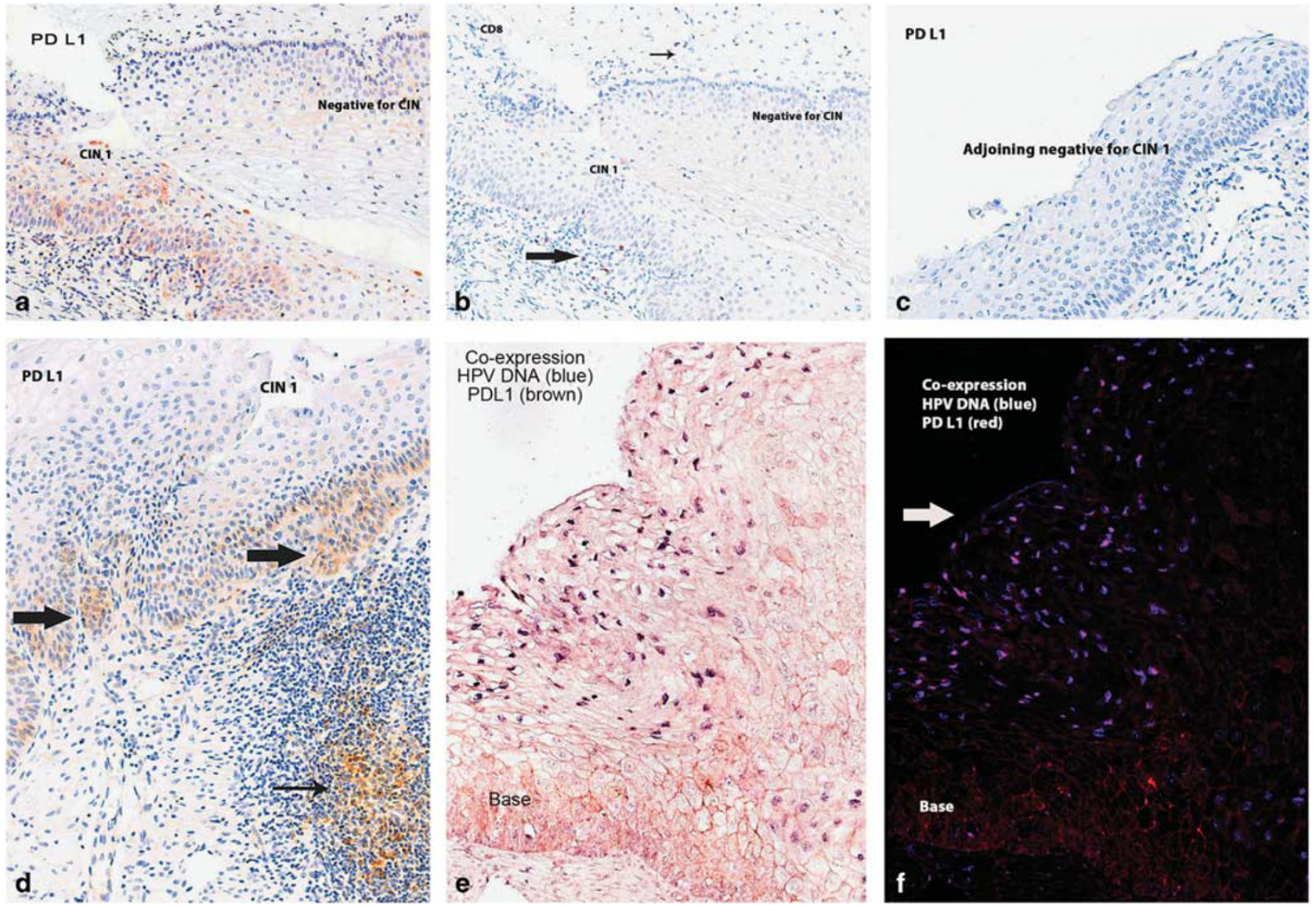

Figure 2 Differential distribution of HPV DNA and PD L1 in cervical tissue. (a) The results of a PD L1 immunohistochemistry test on a cervical biopsy in which the CIN 1 (lower left) and negative for CIN epithelia (upper right) are evident in the same histologic section. Note that a signal is evident toward the base of the lesion in the CIN 1 lesion but not in the adjacent negative for CIN epithelia. CD8 analysis on a serial section shows occasional positive cells in the stroma underlying the CIN 1 (large arrow) but not in the stroma underneath the normal tissue (small arrow, b). (d) Another CIN 1 lesion where the PD L1 signal is strong at the base of the lesion (large arrows) and in collections of mononuclear cells in the adjoining stroma (small arrow). (c) The adjoining histologically unremarkable squamous epithelia where no PD L1 signal is seen in either the epithelia or stroma. (e) The co-expression analysis in the CIN 1 part of this same lesion where the HPV DNA signal (blue) was colocalized with PD L1 (brown). Note that the two signals are present in the same area but in histologically distinct regions; viral DNA is present toward the surface (e) and PD L1 in the cells toward the base. (f) The same section analyzed by the Nuance system in which the lack of fluorescent yellow in the mixed image shows that PD L1 is present in viral infected cells that do not contain large amounts of viral DNA (arrow, surface of lesion).

mononuclear cell(s) were expressing PD L1 in the cervical lesions? Co-expression analysis in the cervical cancers showed that the majority of the PD L1 mononuclear stromal cells were CD8 cells with a smaller proportion being CD68 cells (data not shown). Indeed, basically all of the PD L1 expressing mononuclear cells seen infiltrating the nests of squamous cell cancers were CD8+ (data not shown).

Although CIN 1-2 lesions tend to have more viral DNA per cell than cervical squamous cell cancers, the HPV copy number tends to be very variable in a given lesion. ${ }^{4,5}$ Thus, it can be difficult to correlate the HPV DNA copy number with PD L1 expression. In this regard, we compared the expression of PD L1 in three cervical cancer cell lines with constant and very disparate HPV DNA copy numbers in a blinded fashion. When unblinded to the cell type, it was evident that the Caski cells (about 600 HPV DNA/ cell), HeLa cells (20 copies HPV DNA/cell), and the
SiHa cells (1 HPV DNA/cell) each contained equivalent amounts of PD L1 (data not shown).

\section{Discussion}

The primary finding of this study is that histologically evident infection by human papillomavirus in the cervix was strongly associated with PD L1/PD 1 expression that was found in both the dysplastic/ neoplastic squamous cells and adjoining CD8 mononuclear cells. The baseline expression of PD L1/PD 1 was extremely low even in histologically 'negative for CIN' cervical epithelia from women with HPV infection suggesting that productive viral infection was necessary to induce PD L1 expression. CINs and cervical cancer epithelia each show abundant early ORF HPV expression but only the former shows high L1/L2 expression. ${ }^{1-5,7,8}$ Thus, one can speculate that 

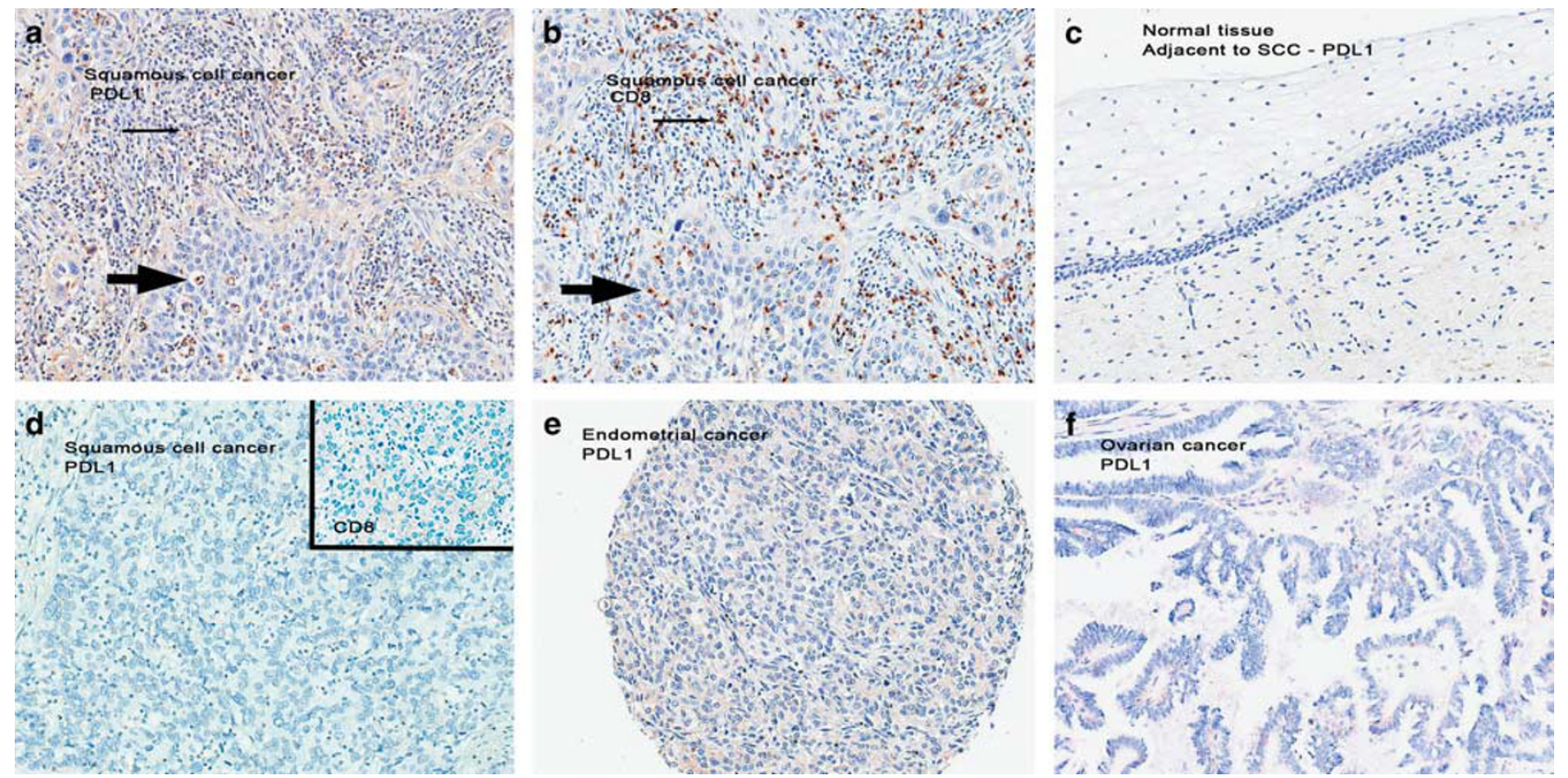

Figure 3 Distribution of PD L1 in the common gynecologic malignancies (a) shows the invasive front of a cervical squamous cell cancer where the nests of cancer cells (large arrow) abuts the adjacent stroma (small arrow). Note the many mononuclear cells that express PD L1 both in the malignant epithelia and stroma. Serial section analysis for CD8 (b) shows that cytotoxic T cells show the same distribution as PD L1. Analysis of adjacent unremarkable squamous epithelia (c) shows the absence of PD L1. (d) A different squamous cell cancer that was negative for PD L1; the insert shows the lack of CD8 cells in the serial section. (e and f) are representative examples of an adenocarcinoma of the endometrium and ovary, respectively. Note the absence of PD L1 production.

certain viral early ORFs may be driving the PD L1 expression that will require additional investigation. Consistent with this observation was that coexpression analysis showed that PD L1 in the CINs was found toward the basal layer, where E6 and E7 RNAs are relatively abundant, and not in the koilocytes, where other early ORFs and L1/L2 RNAs dominate. ${ }^{7}$ HPV high risk E6 and E7 RNA are abundant in squamous cell cancer of the cervix. ${ }^{8}$ The equivalent detection of PD L1 in Caski and SiHa cells does suggest that viral DNA levels are not inducing PD L1 levels. These two cell lines, however, do show equivalent HPV E6/E7 expression. ${ }^{4}$ The finding that only cervical cancer, and not endometrial or ovarian carcinoma, was strongly associated with PD L1 expression is consistent with the hypothesis that HPV infection may enhance PD L1 production.

The PD L1 positive mononuclear cells were invariably present in the same histologic area as the dysplastic/neoplastic squamous cells also making this protein. Serial section and co-expression analysis showed that most of the mononuclear cells that were making PD L1 were CD8 cells. It has been documented by other researchers that CD8 cells are the dominant lymphocytic cell type in cervical carcinoma $^{20}$ and that CD8 cells may have a role in viral clearance in regressing CINs. ${ }^{11}$ Our results are consistent with a key role of CD8 cytotoxic cells in cervical precancer/cancer and suggest that the CD8 activity may be reduced due to the high expression of PD L1. Indeed, we noted that a relatively low percentage of the CD8 cells evident in the CIN lesions and the cervical squamous cell carcinomas were activated, based on low Ki67 indexes, and low coexpression of RANTES plus granzyme B. Our result that $\mathrm{T}$ cells are the primary source of PD L1 in high risk HPV infection is consistent with the study of Yang et $a l^{21}$ although they studied exfoliated cervical cells compared with the formalin fixed paraffin embedded tissues employed in this study.

Recently, Herbst et al demonstrated that paralleled increase expression of CD8 and PD L1 was evident in a variety of stage IV carcinomas, including lung, colorectal, and renal cell. They noted elevated PD L1 expression in mononuclear cells coincident with increased expression of the protein by cancer cells in about $1 / 3$ of such cases. Importantly, they found that increased CD8/PD L1 expression predicted response to anti- PD L1 therapy. ${ }^{15}$ Tumeh et al arrived at similar conclusions in metastatic melanomas. ${ }^{16}$ Our results demonstrate that cervical cancers show a much higher percentage of cases with increased PD L1 and CD8 production than the tumors included in these two studies, ${ }^{15,16}$ even using a twofold higher threshold and, thus, may be especially amenable to anti-PD L1 therapy, which will require additional investigation. Further, the much higher degree of expression of PD L1 in the cervical cancers compared to the tumors studied by Herbst et al and Tumeh et al is consistent with the theory that it is the virus HPV as well as the cancer associated foreign 

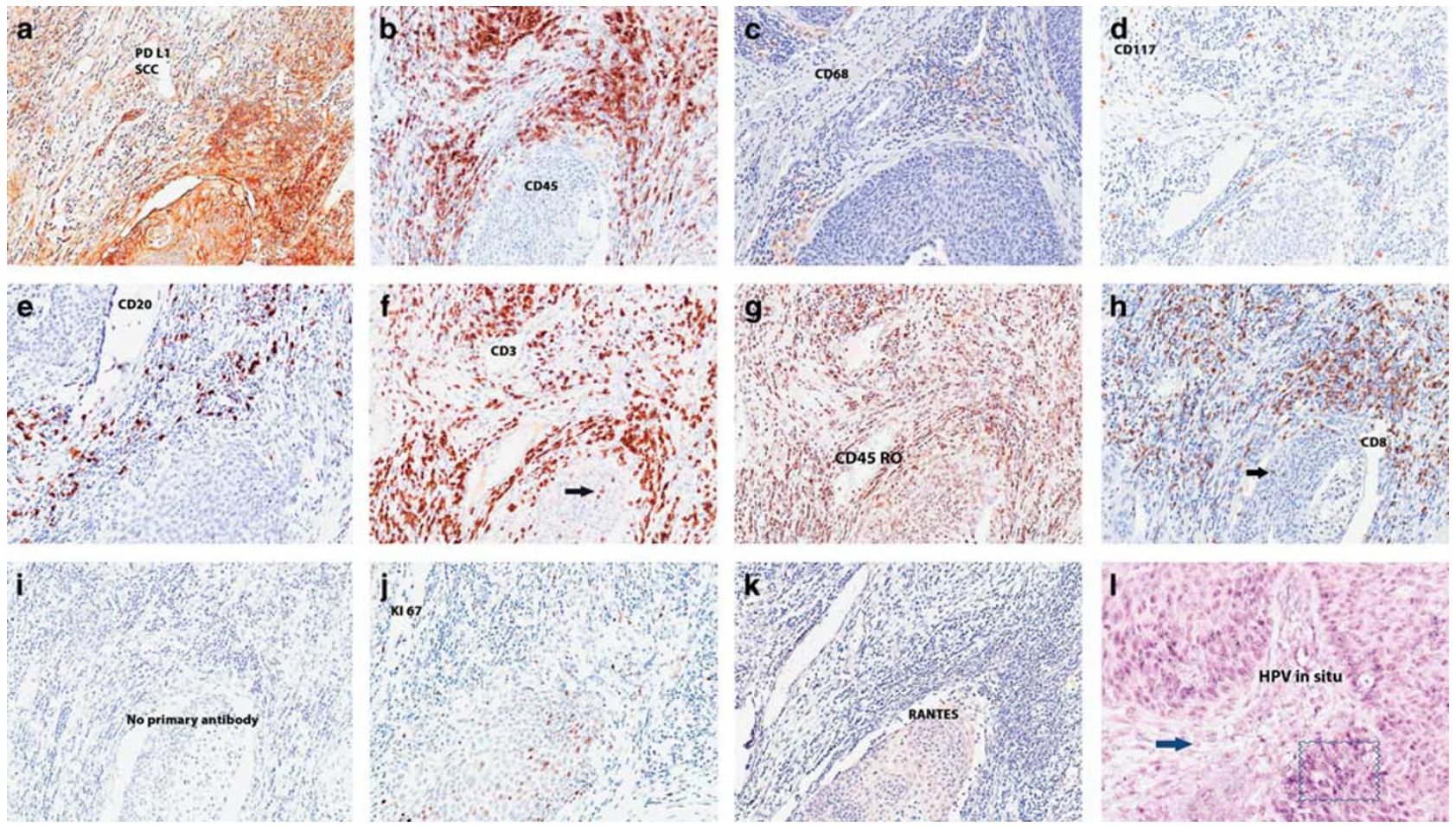

Figure 4 Serial section analysis of the immune response in a cervical squamous cell cancer. The invasive front of a cervical squamous cell cancer is depicted through twelve sections. The PD L1 immunohistochemistry analysis (a) shows strong expression in both the invasive squamous cancer cells (middle lower part of panel) and the surrounding stromal cells. The recruitment of lymphocytes as seen by the CD45 test is strong (b), which co-exists with the macrophage (CD68; (c)) and the NK cell response ((d); CD 117). Subdivision of the lymphocyte response shows a B cell response ((e); CD20) and a stronger T-cell response ((f); CD3). Note that only the T cells are seen infiltrating the malignant squamous cells (arrow). The CD45RO response, indicative of T-cell activation, is strong (g) as is the cytotoxic T-cell response ((h); CD8); note the CD8 cells infiltrating the tumor nests (arrow). (i) is the negative control (omission of the primary antibody). Despite the high CD8 numbers, note the evidence of inhibited activation of the cytotoxic T cells as seen as a weak Ki67 response in the CD8+ stromal cells (j) and the rare cells in this area that express RANTES (k). (l) The HPV in situ hybridization; note the punctate signal in the malignant epithelia (boxed area) and the lack of a signal in the stromal cells (arrow). NK, natural killer.

antigens that may be driving the PD L1/PD 1/CD8 axis. This, in turn, suggests that oncolytic virus therapy directed against cancers may likewise augment the PD L1/CD8 axis and make the tumors more accessible to anti- PD L1 therapy.

Karim et al studied 115 cervical cancers and found that PD L1 was detected in 19\% of cancers and noted that PD L1 and Treg co-infiltration was associated with improved survival. ${ }^{22}$ The study ${ }^{22}$ analyzed PD L1 expression only on tumor cells. Our study and others suggest that tumor infiltrating CD8 cells may be the primary source of PD L1 in cancers ${ }^{15,16}$ including cervical cancer. Still, our data and that of Karim et al underscore the presence and importance of checkpoint inhibitors such as PD L1 and Tregs in the biology of cervical cancer.

Histologic diagnosis of CIN is not straightforward. Many investigators have indicated that re-examination of CINs can show false negative and, more commonly, false positive readings of over $25 \% .{ }^{23}$ As cervical HPV infection is a sexually transmitted disease, the emotional implications of a false positive diagnosis to the woman may be severe. Thus, there has been a concerted effort to find biomarkers that can differentiate HPV infection of the cervix from its mimics that include p16, MIB-1, HPV L1/L2, and others. ${ }^{1-5}$ The results in this study suggest that PD L1 expression may be able to differentiate productive HPV infection, which typically is treated with ablative therapy, from its mimics, which do not require therapy. Clearly, this hypothesis will require much larger studies as the focus of this paper was PD L1 expression in a wide variety of gynecologic lesions and cancers, not just CIN.

It is well established that HPV infection of the cervix can spontaneously regress. ${ }^{1-5,9,10}$ Most of these studies are based on the detection of HPV DNA by the ultra-sensitive hybrid capture method or viral DNA by polymerase chain reaction (PCR) plus cytologic abnormalities on the Pap smear. As Hybrid Capture or PCR cannot differentiate between latent and productive HPV infection, it is not clear what percentage of productive infections (that is, classic histologically defined CINs) actually regress. Regardless, it is clear that in about $50 \%$ of cases of HPV positivity by a solution phase highly sensitive method and an abnormal Pap smear the HPV type 
will no longer be detected within 6-12 months. ${ }^{9,10}$ In addition, it has been shown that ablation of a CIN lesion leads to the elimination of the specific HPV type responsible for the lesion in $\sim 85 \%$ of cases and that, if the lesion does recur, it will be due to a different HPV type. ${ }^{12}$ Clearly, this data suggests an effective HPV type specific immune clearance mechanism in the cervix, which is the basis of the highly effective vaccines against HPVs $6,11,16$, and 18. ${ }^{13,14}$ Our study did not address the issue of regressing CINs. However, the strong correlation of PD L1 and CIN lesions does raise the possibility that this protein may have a role in the spontaneous clearance of HPV infections.

In sum, this study shows that infection by the common sexually transmitted DNA virus HPV is strongly associated with PD L1 expression that is found in the dysplastic squamous cells, typically toward the base of the CIN, as well as in the malignant squamous cells of cervical cancer, and that this is strongly associated with possible 'crosstalk' production of PD L1 in the CD8 cells that are recruited to the area of viral infection. The mechanism of PD L1 upregulation by the viral infection will require further study. Possible mechanism would include direct HPV RNA inducement of PD L1, HPV dysregulation of microRNA expression patterns ${ }^{24}$ that can, in turn, affect PD L1 production, and more general signals of viral infection such as, for example, production of interferon gamma. Regardless of the mechanism, the possible significance of these findings, which need to be addressed include using PD L1 as a biomarker of productive HPV infection and directing treatment against PD L1 to eradicate HPV infection and the associated lesion, especially in women with squamous cell cervical cancer.

\section{Acknowledgments}

We greatly appreciate the excellent computer/ photomicroscopy assistance of Dr Margaret Nuovo, the computer graphic assistance by Sandra Sue Otera, and the financial support of the LEWIS FOUNDATION.

\section{Disclosure/conflict of interest}

The authors declare no conflict of interest.

\section{References}

1 zur Hausen H. Papillomaviruses causing cancer: evasion from host-cell control in early events in carcinogenesis. J Natl Cancer Inst 2000;92:690-698.

2 Bosch FX, Lorincz A, Munoz N et al. The causal relation between human papillomavirus and cervical cancer. J Clin Pathol 2002;55:244-265.

3 Stoler MH. The virology of cervical neoplasia: an HPVassociated malignancy. Cancer J 2003;9:360-367.
4 Nuovo GJ. The role of HPV in gynecologic diseases: an update. Critical Reviews in Clin Lab Sciences 2000;37:183-215.

5 Nuovo GJ. Low grade Squamous Intraepithelial Lesions. In: Nuovo GJ. Cytopathology of the female genital tract: An integrated approach. Williams and Wilkins: Baltimore, 1993, pp 155-214.

6 Chen EY, Tran A, Raho CJ et al. Histological 'progression' from low (LSIL) to high (HSIL) squamous intraepithelial lesion is an uncommon event and an indication for quality assurance review. Mod Pathol 2010;23:1045-1051.

7 Crum CP, Nuovo GJ, Friedman D et al. Accumulation of RNA homologous to human papillomavirus type 16 open reading frames in genital precancers. J Virol 1988;62:84-90.

8 van den Brule AJ, Cromme FV, Snijders PJ et al. Nonradioactive RNA in situ hybridization detection of human papillomavirus 16-E7 transcripts in squamous cell carcinomas of the uterine cervix using confocal laser scan microscopy. Am J Pathol 1991;139: 1037-1045.

9 Nobbenhuis MA, Helmerhorst TJ, van den Brule AJ et al. Cytological regression and clearance of high-risk human papillomavirus in women with an abnormal cervical smear. Lancet 2001;58:1782-1783.

10 Paraskevaidis E, Arbyn M, Sotiriadis A et al. The role of HPV DNA testing in the follow-up period after treatment for CIN: a systematic review of the literature. Cancer Treat Rev 2004;30:205-211.

11 Trimble CL, Clark RA, Thoburn C et al. Human papillomavirus 16-associated cervical intraepithelial neoplasia in humans excludes CD8 T cells from dysplastic epithelium. J Immunol 2010;185:7107-7114.

12 Nuovo GJ, Pedemonte BA. Human papillomavirus types and recurrent genital warts. JAMA 1990;263: 1223-1226.

13 Herweijer E, Leval A, Ploner A et al. Association of varying number of doses of quadrivalent human papillomavirus vaccine with incidence of condyloma. JAMA 2014;311:597-603.

14 Villa LL, Ault KA, Giuliano AR et al. Immunologic responses following administration of a vaccine targeting human papillomavirus Types 6, 11, 16, and 18. Vaccine 2006;24:5571-5583.

15 Herbst RS, Soria JC, Kowanetz M et al. Predictive correlates of response to the anti-PD-L1 antibody MPDL3280A in cancer patients. Nature 2014;515:563-567.

16 Tumeh PC, Harview CL, Yearley JH et al. PD-1 blockade induces responses by inhibiting adaptive immune resistance. Nature 2014;515:568-571.

17 Xie Z, Chen Y, Zhao S et al. Intrahepatic PD-1/PD-L1 up-regulation closely correlates with inflammation and virus replication in patients with chronic HBV infection. Immunol Invest 2009;38:624-638.

18 Nuovo GJ. The basics of in situ hybridizationIn:Nuovo GJIn situ Molecular Pathology and Co-expression analyses. Elsevier: San Diego, CA, 2013, pp 81-132.

19 Nuovo GJ, Garofalo M, Valeri N et al. Reovirusassociated reduction of microRNA-let-7d is related to the increased apoptotic death of cancer cells in clinical samples. Mod Pathol 2012;25:1333-1344.

20 Monnier-Benoit S, Mauny F, Riethmuller D et al. Immunohistochemical analysis of CD4+ and CD8+ T-cell subsets in high risk human papillomavirusassociated pre-malignant and malignant lesions of the uterine cervix. Gynecol Oncol 2006;102:22-31. 
21 Yang W, Song Y, Lu YL et al. Increased expression of programmed death (PD)-1 and its ligand PD-L1 correlates with impaired cell-mediated immunity in high-risk human papillomavirus-related cervical intraepithelial neoplasia. Immunology 2013;139: 513-522.

22 Karim R, Jordanova ES, Piersma SJ et al. Tumorexpressed B7-H1 and B7-DC in relation to PD-1+ T-cell infiltration and survival of patients with cervical carcinoma. Clin Cancer Res 2009;15:6341-6347.

23 Richart RM, Nuovo GJ. HPV-DNA in situ hybridization can be used for the quality control of diagnostic biopsies. Obstet Gynecol 1990;75:223-226.

24 Wang X, Wang HK, Li Y et al. microRNAs are biomarkers of oncogenic human papillomavirus infections. Proc Natl Acad Sci USA 2014;111:4262-4267. 